\title{
Electroactive carbon nanoforms: a comparative study via sequential arylation and click chemistry reactions $\nmid \ddagger$
}

\author{
Jaime Mateos-Gil, ${ }^{\S a}$ Laura Rodríguez-Pérez, ${ }^{\S a}$ María Moreno Oliva, ${ }^{\S b}$ Georgios Katsukis, ${ }^{b}$ Carlos \\ Romero-Nieto, ${ }^{b, c} \mathbf{M}^{\mathrm{a}}$ Ángeles Herranz, ${ }^{a}$ Dirk M. Guldi ${ }^{b}$ and Nazario Martín ${ }^{* a, d}$
}

\author{
s Received (in $X X X, X X X)$ Xth $X X X X X X X X X 20 X X$, Accepted $X$ th $X X X X X X X X X 20 X X$ \\ DOI: 10.1039/b000000x
}

The reactivity of several carbon nanoforms (CNF), single-walled carbon nanotubes (SWCNT), multiwalled carbon nanotubes (MWCNT) and graphene, has been investigated through a combination of arylation and click chemistry $\mathrm{Cu}^{\mathrm{I}}$-mediated azide-alkyne cycloaddition (CuAAC) reactions. The approach 10 is based on the incorporation of electroactive $\pi$-extended tetrathiafulvalene (exTTF) units into the triazole linkers to modulate the electronic properties of the obtained conjugates. The introduction of strain, by bending the planar graphene sheet into a 3D carbon framework, is responsible of the singular reactivity observed in carbon nanotubes. The formed nanoconjugates were fully characterized by analytical, spectroscopic, and microscopic techniques (TGA, FTIR, Raman, UV-Vis-NIR, cyclic voltammetry, TEM 15 and XPS). In the case of SWCNT conjugates, where the functionalization degree is higher, a series of steady-state and time resolved spectroscopy experiments revealed a photoinduced electron transfer from the exTTF unit to the electron-accepting SWCNT.

\section{Introduction}

With the advent of nanoscience and nanotechnology, carbon 20 nanoforms, including fullerenes, carbon nanotubes, and graphene, have become the most popular nanomaterials due to their excellent mechanical, electronic, and thermal properties. ${ }^{1}$ In particular, the successful isolation of single-layer graphene in 2004 caused a worldwide revolution aiming at the utilization of 25 its unique structural and electronic properties in fields such as nanoelectronics, biosensors, supercapacitors, catalysis, nanomedicine, intercalation materials or polymer composites just to name a few. ${ }^{2}$ Likewise, the appealing features of fullerenes ${ }^{3}$ and carbon nanotubes $(\mathrm{CNT})^{4}$ also make them attractive building 30 blocks for the aforementioned applications.

Two major drawbacks hamper, however, the application of CNT and graphene for practical purposes, namely their lack of solubility in common solvents and their relative inert surfaces. To overcome such setbacks, both covalent and non-covalent 35 chemical modifications have been investigated in recent years. ${ }^{5}$ On one hand, the non-covalent functionalization of CNT and graphene endows them with satisfactory solubility, while conserving their major structural characteristics. In most cases, the stability of the conjugates is, nevertheless, strongly influenced 40 by the experimental conditions (solvents, $\mathrm{pH}$, temperature, etc.). ${ }^{6}$ The covalent functionalization, on the other hand, results in stable products, but implies the saturation of some of the $\mathrm{sp}^{2}$ carbons of the CNFs' framework. ${ }^{7}$ As a matter of fact, the electronic properties are accordingly impacted.

45 At the fundamental level, the chemical reactivity of $\mathrm{sp}^{2}$ hybridized carbons is affected by the curvature, which alters the distribution of electron density in graphene and CNT. The chemical inertness of graphene can be easily understood on the basis of its planar framework, although its reactivity increases at ${ }_{50}$ the edges compared with the basal plane. For CNT, a direct correlation between tube diameter and reactivity exists. The pyramidalization angle among carbon bonds, that is, the difference between the sigma-pi orbital angle and $90^{\circ}$, and the $\pi$ orbital misalignment between carbon atoms decrease as the ${ }_{55}$ diameter increases, rendering small-diameter CNT more reactive than large-diameter $\mathrm{CNT}^{8}$

Despite the fact that the covalent chemistry of carbon nanoforms has been intensively probed, to date, only a few studies focus on the comparative assays regarding these carbon 60 frameworks. ${ }^{9}$ Considering, for example, the basic details of chemical reactivity regarding either flat or curved $\mathrm{sp}^{2}$-hybridized carbon materials different consequences should stem from their chemical functionalization.

The thrust of the present study is to perform a systematic and ${ }_{65}$ comparative analysis between the general reactivity of different types of CNT and graphene in light of benchmark reactions, which allows evaluating the properties of the different products. The reaction of all CNF with diazonium salts of aromatic compounds, bearing also alkyne functional groups, yields directly 70 arylated derivatives that further react in a click process. Specifically, the combination of arylation and $\mathrm{Cu}$-catalyzed Huisgen 1,3-dipolar cycloaddition of azides and alkynes (CuAAC) is widely applicable in organic and supramolecular chemistry and has recently been employed with great success in the 75 area of CNT and graphene chemistry. ${ }^{10}$ In addition, $\pi$-extended tetrathiafulvalenes $\quad(9,10$-bis(1,3-dithiol-2-ylidene)-9,10-dihydro- 
anthracene, exTTF) are powerful electron donors with have been successfully used for preparing new photoinduced electron transfer systems and solar energy conversion devices. ${ }^{11}$ exTTF has been widely used to form electroactive architectures when $s$ linked to fullerenes and CNTs. Importantly, efficient electronic communication between the carbon nanoforms and exTTF has been documented for the ground and excited states by means of steady-state as well as time-resolved spectroscopies. ${ }^{12,13}$

\section{Results and discussion}

10 The synthesis of the different CNF-exTTF conjugates is described in Scheme 1. Note that the representation provides only a simplified view of the starting materials, namely SWCNT, MWCNT, and graphene. Following a previously reported procedure, ${ }^{14}$ exTTFs were endowed with azido groups, which

15 were subjected to a click chemistry reaction with the terminal ethynyl groups of the desired carbon nanostructures.
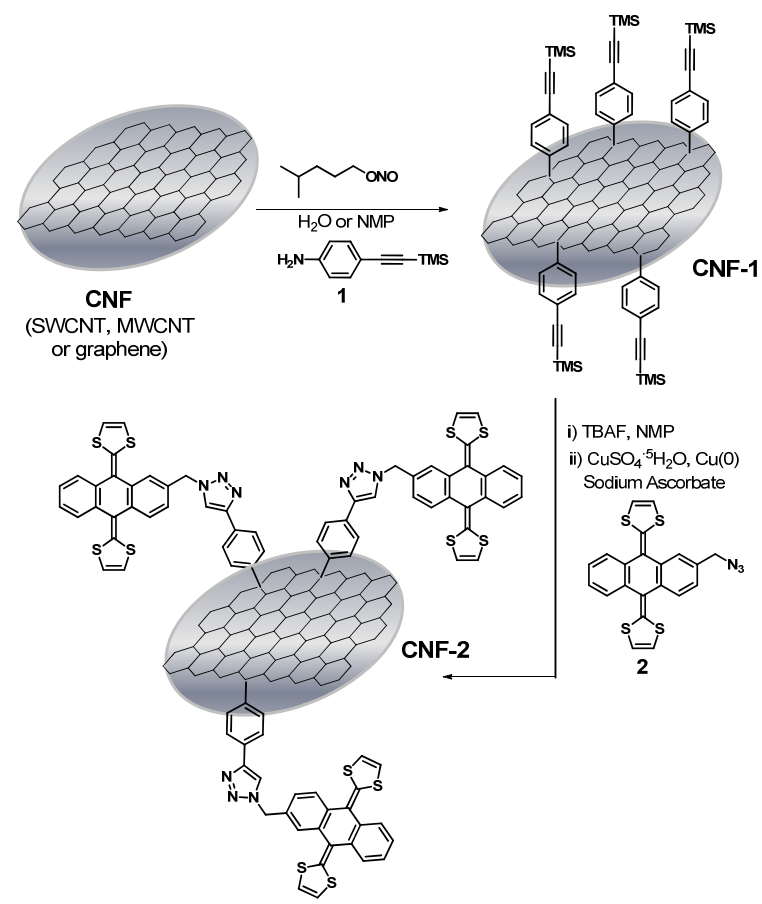

Scheme 1. Synthetic route towards functionalized CNF considering Tour conditions (CNF-1) and the click chemistry CuAAC protocol (CNF-2).

20 Regarding the introduction of ethynyl functionalities into the CNFs, the $\pi$-electronic systems of SWCNT, MWCNT and graphene were covalently modified via aryl diazonium chemistry. ${ }^{15}$ This radical addition reaction affords intermediates that are decorated with phenylethynyl groups, when 425 [(trimethylsilyl)ethynyl]aniline is used as starting material. SWCNTs and MWCNTs were reacted following the well stablished procedure reported by Tour et al. ${ }^{16}$ using water as reaction medium, in the presence of isoamyl nitrite and 4[(trimethylsilyl)ethynyl]aniline, affording SWCNT-1 and

30 MWCNT-1. This functionalization method has been slightly modified when using graphene as the starting nanocarbon. To this end, the pristine graphene material was obtained through graphite exfoliation in $N$-methylpyrrolidone (NMP) following Coleman's procedure toward few-layered graphene suspensions with high
${ }_{35}$ degree of purity. To simplify the discussions we will keep referring from here on to graphene. ${ }^{17}$ Such a graphene suspension in NMP was immediately reacted with 4-[(trimethylsilyl)ethynyl] aniline and isoamyl nitrite, which formed in situ the corresponding aryl diazonium salt, and, which yielded the 40 covalent graphene conjugates. Notably, this process was followed by a second cycle of the same Tour reaction to increase the number of functional groups anchored onto the basal plane of graphene (GR-1).

Deprotection of the alkyne groups with tetrabutylammonium 45 fluoride (TBAF) and subsequent $\mathrm{Cu}(\mathrm{I})$-catalyzed 1,3-dipolar cycloaddition reaction with azido exTTF 2 was carried out in situ under thermal conditions for the three carbon nanoconjugates affording SWCNT-2, MWCNT-2 and GR-2.

The protected alkyne groups anchored on the three 50 intermediate carbon nanoforms skeletons were readily identified by Fourier transform infrared spectroscopy (FTIR). The stretching mode of the acetylenic spacer, $v(C \equiv C)$, appears as a weak band around $2200-2100 \mathrm{~cm}^{-1}$, a region where very few organic compounds show IR-active modes. ${ }^{18}$ The appearance of this 55 band at $2159 \mathrm{~cm}^{-1}$ for SWCNT-1 (Figure S1), at $2156 \mathrm{~cm}^{-1}$ for MWCNT-1 (Figure 1), and at $2165 \mathrm{~cm}^{-1}$ for GR-1 (Figure S2) corroborates the presence of alkynes on the corresponding surfaces. These bands disappear after CuAAC reaction with azido-exTTF 2 for all of the carbon nanostructures, that is,

${ }_{60}$ SWCNT-2, MWCNT-2, and GR-2. This is due to the formation of triazole rings. The characteristic bands of the skeletal in-plane vibration is seen at $1599 \mathrm{~cm}^{-1}$ for SWCNT, at $1580 \mathrm{~cm}^{-1}$ for MWCNT, and at $1583 \mathrm{~cm}^{-1}$ for graphene. ${ }^{19}$ As a complement, the aliphatic $\mathrm{C}-\mathrm{H}$ stretching mode located between 2850 and 2950 ${ }_{65} \mathrm{~cm}^{-1}$ and the $\mathrm{C}-\mathrm{H}$ bending mode at around $1250-1500 \mathrm{~cm}^{-1}$ are discernable in all the intermediate and final nanoconjugates.

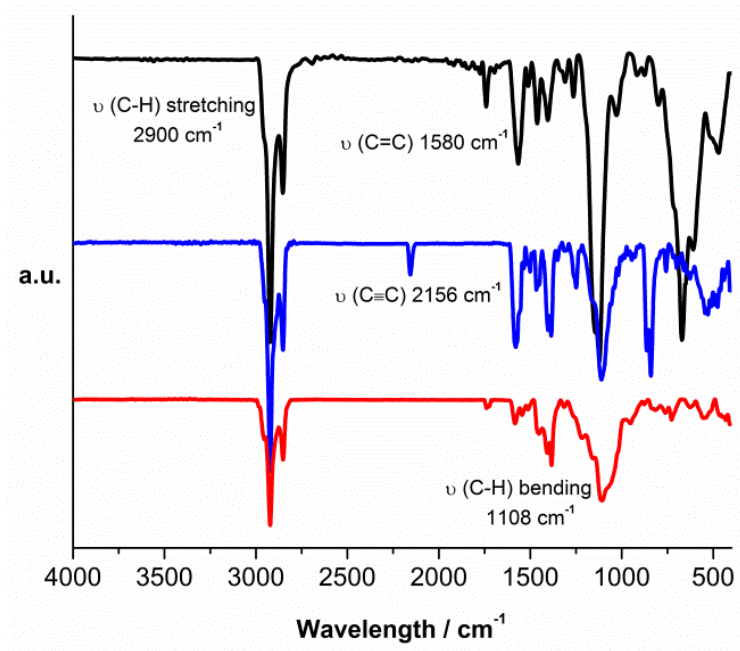

Figure 1. FTIR spectra of MWCNT-2 (red), MWCNT-1 (blue), and pristine MWCNT (black).

70 While FTIR spectroscopy reveals important chemical information regarding the incorporated functional groups, Raman spectroscopy gives insights into the surface functionalization of SWCNT, MWCNT, and graphene. ${ }^{20}$ Upon $785 \mathrm{~nm}$ excitation of pristine SWCNT, SWCNT-1, and SWCNT-2 the three most 75 important signatures of these hollow cylinders are noted. Firstly, the radial breathing modes bands (RBM) between 186 and 270 
$\mathrm{cm}^{-1}$ suggest the presence of both metallic and semiconducting SWCNTs with a range of diameters between 0.8 and $1.4 \mathrm{~nm}$. Secondly, the D mode is located between $1290 \mathrm{~cm}^{-1}$ and $1300 \mathrm{~cm}^{-1}$. Finally, the G mode or MT-tangential mode is seen between $51592-1595 \mathrm{~cm}^{-1}$ (Figure S3). Upon covalent functionalization the $\mathrm{D}$ band increases due to the re-hybridization of carbon atoms from $\mathrm{sp}^{2}$ to $\mathrm{sp}^{3}$. Importantly, the $\mathrm{I}_{\mathrm{D}} / \mathrm{I}_{\mathrm{G}}$ ratio (Table inserted in Figure S3) is used to quantify the amount of covalently anchored groups. The latter increases from 0.064 for pristine SWCNT to 100.283 for SWCNT-1. For SWCNT-2, the $\mathrm{I}_{\mathrm{D}} / \mathrm{I}_{\mathrm{G}}$ ratio remains, however, constant with 0.284 . As such, the subsequent click reaction takes only place with the alkyne groups previously anchored to SWCNT. Moreover, the G tangential mode experiences a small up-shift (1-2 nm) going from pristine ${ }_{15}$ SWCNT to SWCNT-1 and SWCNT-2. This is in sound agreement with previous findings regarding the non-covalent modification of SWCNT with exTTF-based receptors. ${ }^{13 b, 21}$

In contrast to SWCNTs, the intensity ratio between the D and G bands for MWCNTs increases only slightly from MWCNT to 20 MWCNT-2, although the broader G band includes contributions from disorder, defects, or intercalation between CNT walls (Figure S4). ${ }^{22}$
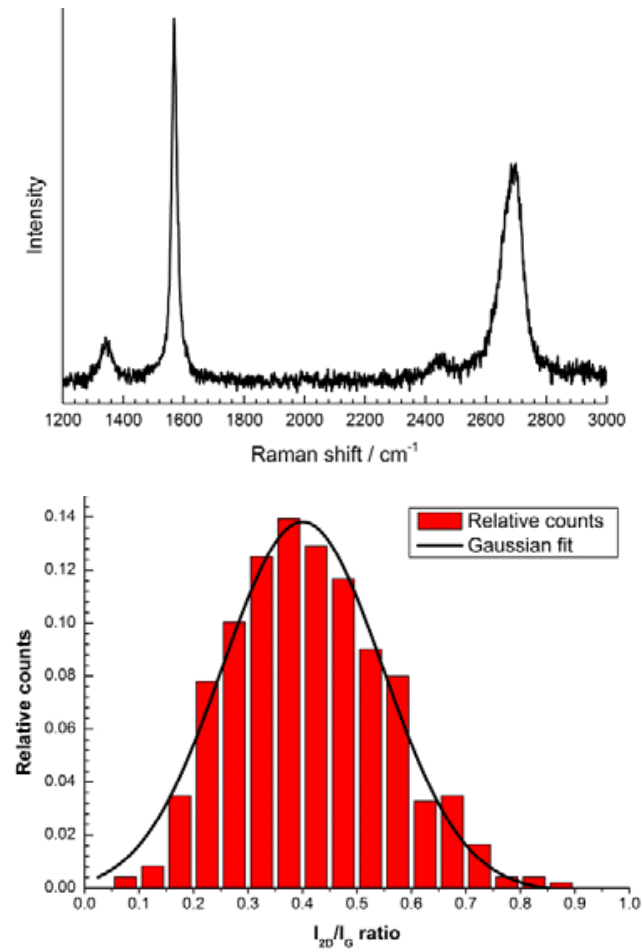

25 Figure 2. Raman spectrum of GR-2 on top. Bottom, histogram with relative counts vs. $\mathrm{I}_{2 \mathrm{D}} / \mathrm{I}_{\mathrm{G}}$ ratio and the corresponding Gaussian distribution. The sample was drop casted from a DMF dispersion onto $\mathrm{SiO}_{2}$ wafer and was excited at $532 \mathrm{~nm}$.

Graphene samples excited at $532 \mathrm{~nm}$ reveal the typical Raman 30 peaks of exfoliated graphite, namely, the D-, G-, and 2D-bands centered around 1340,1565 , and $2678 \mathrm{~cm}^{-1}$, respectively. Upon exfoliation, a slight increase in the $\mathrm{D}$ band intensity is noticed due to defects induced by the ultrasonication process. A significant change in the shape and intensity of the $2 \mathrm{D}$ peak compared to 35 bulk graphite is observed (Figure S5). The 2D band obtained with full width at half maximum (FWHM) values of $76 \mathrm{~cm}^{-1}$ was fit with 6 different Lorentzian functions with a FWHM of $24 \mathrm{~cm}^{-1}$, which corresponds to graphene. ${ }^{22}$ In addition, functionalization of the basal plane of graphene to form $\mathrm{sp}^{3}$ domains contributes as 40 well to an increase of the D band intensity (Figure S6). In analogy to SWCNT functionalization, graphenic materials present an $\mathrm{I}_{\mathrm{D}} / \mathrm{I}_{\mathrm{G}}$ increase from 0.051 to 0.18 after Tour reaction in GR-1, but remain constant for GR-2. This result, with a much lower difference on the $\mathrm{I}_{\mathrm{D}} / \mathrm{I}_{\mathrm{G}}$ ratio upon functionalization of 45 graphene compared to SWCNT, highlights the lower reactivity of graphene under similar reaction conditions. ${ }^{23}$ Figure 2 shows a typical Raman spectrum of GR-2 and a statistical distribution of $\mathrm{I}_{2 \mathrm{D}} / \mathrm{I}_{\mathrm{G}}$ intensity ratio for a GR-2 suspension drop casted onto $\mathrm{SiO}_{2}$. The statistical distribution of $\mathrm{I}_{2 \mathrm{D}} / \mathrm{I}_{\mathrm{G}}$ is best fit with a ${ }_{50}$ Gaussian distribution function, which peaks at a ratio of 0.4. For bulk graphite or few-to multi-layer graphene the intensity ratio is 0.7 or less. ${ }^{24}$ As such, the Raman spectra prompt to predominantly few-layer graphene, which likely originate from the initial exfoliation step performed with NMP. In sound agreement with 55 the transmission electron microscopy (TEM) measurements vide infra - we concluded that we are dealing, at least upon drop casting, mainly with few-to multi-layer graphene.

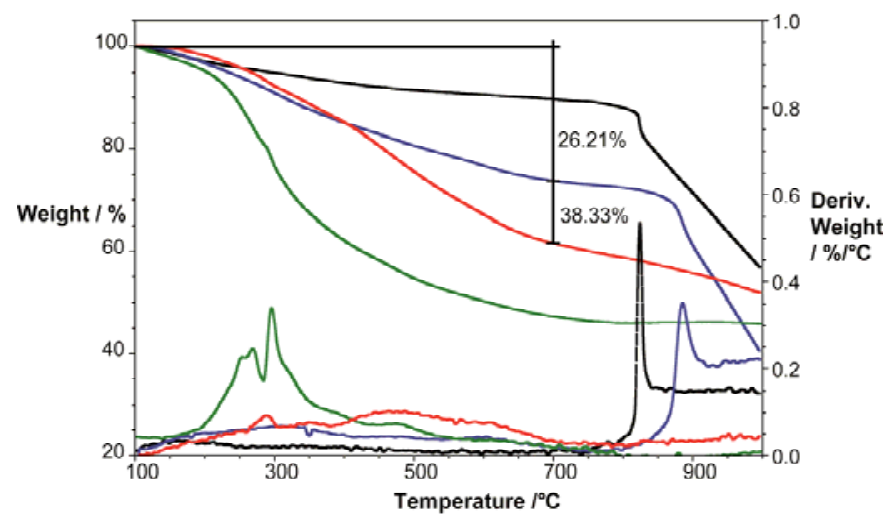

Figure 3. TGA and first derivative curves of pristine SWCNT (black), 60 nanoconjugates SWCNT-1 (blue) and SWCNT-2 (red), together with the thermal decomposition of the exTTF molecule 2 (green) recorded under inert conditions.

To estimate the grafting density, thermogravimetric analysis (TGA) measurements were performed. A significantly higher ${ }_{65}$ degree of functionalization was obtained for SWCNT-1 (Figure 3) when compared to MWCNT-1 (Figure S7) and GR-1 (Figure S8) under similar reaction conditions. More specifically, the weight loss measured for the thermal decomposition of the phenylacetylene groups in SWCNT-1 was $26 \%$ compared to $8 \%$ 70 for MWCNT-1 and $14 \%$ for GR-1. As expected, the carbon nanostructure reactivities were found to depend on their topography. A higher curvature in, for example, SWCNTs renders them more favorable for covalent chemical reactions than MWCNT or graphene sheets. Moreover, the linkage of the 75 electron-donor exTTF via click reaction increases the observed weight loss. The total decomposition due to the SWCNT-2 functionalization was $38 \%$, which refers to a $12 \%$ increase compared to SWCNT-1. From this we estimate 1 functional group per $72 \mathrm{C}$ atoms. For MWCNT-2, the weight loss is $21 \%$ $80(29.6 \%$ total weight loss), which corresponds to 1 functional group per $106 \mathrm{C}$ atoms. For GR-2, the weight loss of $6.9 \%(21.3$ $\%$ total weight loss) corresponds to 1 functional group per $168 \mathrm{C}$ 
atoms (see Figure S9 for a direct comparison on the TGA curves of the three functionalized CNF-2). ${ }^{25}$ In summary, TGA measurements provided first insights into the efficiency of the click reaction and suggested that not all phenylacetylene groups 5 were further functionalized through the copper catalyzed click reaction.

X-ray photoelectron spectroscopy (XPS) further demonstrated the successful functionalization of the carbon nanoforms. The spectra of SWCNT-2 (Figure S10), MWCNT-2 (Figure 4), and

${ }_{10}$ GR-2 (Figure S11) display the spectroscopic signatures of carbon, oxygen, nitrogen, sulfur, and silicon. Upon treatment with 4-(2-trimethylsilyl)ethynylaniline and isoamyl nitrite, a significant amount of Si $2 p$ was detected in the XPS spectra for SWCNT-1, MWCNT-1, and GR-1, stemming from the alkyne 15 protecting group after Tour reaction. An important observation is the lack of nitrogen in the intermediate samples, confirming the absence of unreacted species, which were removed during the work-up. In stark contrast, nitrogen is discernible in SWCNT-2, MWCNT-2, and GR-2 samples. The deconvolution of the C1s 20 energy level signals was accomplished with five GaussianLorentzian curves, which were attributed to the various carbon atoms $\left(\mathrm{C} \mathrm{sp}, \mathrm{C} \mathrm{sp}{ }^{2}\right.$ and $\mathrm{C} \mathrm{sp}{ }^{3}, \mathrm{C}=\mathrm{O}$, and $\left.\mathrm{C}-\mathrm{O}\right)$ present in the carbon nanoforms (Figure 4, inserted). ${ }^{26}$ The deconvolution of the $\mathrm{N}$ 1s energy level signal, for SWCNT-2, MWCNT-2, and GR-2 25 let to two peaks. For MWCNT-2 (Figure 4), the first peak located at $401.5 \mathrm{eV}$ is attributed to one nitrogen atom of the triazole ring, while the other two nitrogen atoms give rise to the second peak at $400.1 \mathrm{eV} .{ }^{27} \mathrm{An}$ analogous behavior was observed for SWCNT-2 and GR-2 (Figures S10 and S11). The absence of 30 the peak corresponding to the free azide groups at $405.0 \mathrm{eV}$ unambiguously proves that the azido exTTF is covalently attached to the carbon nanomaterials rather than beeing simply physisorbed onto the surface by $\pi$-stacking interactions. ${ }^{28} \mathrm{~A}$ single S $2 p$ signal at around $170 \mathrm{eV}$ was found for the samples 35 subjected to the click reaction, indicating the presence of sulfur from the organic electron-donor. In line with the results discussed for TGA - vide supra - the Si $2 p$ peak is still observable for SWCNT-2, MWCNT-2, and GR-2. Nevertheless, its atomic percentage indicates an important decrease compared to the 40 amount of $\mathrm{Si}$ observed in the intermediate products, suggesting a non-complete deprotection of the alkyne group and, therefore, a non-quantitative click reaction.

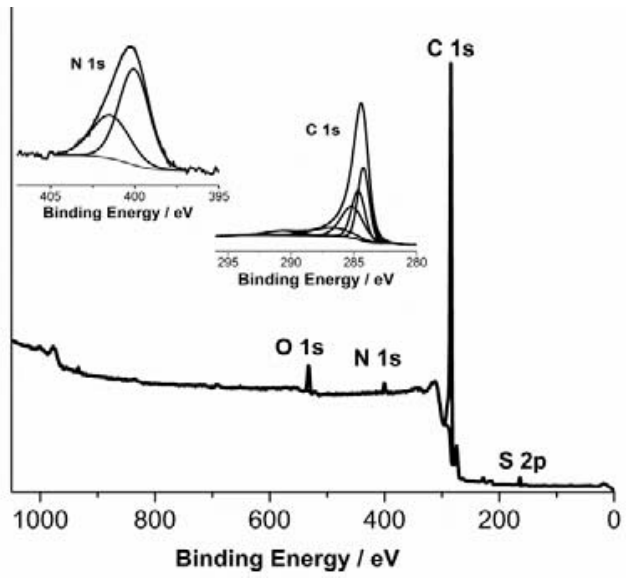

Figure 4. XPS spectrum of MWCNT-2 and deconvoluted XPS core level 45 spectra of carbon $\mathrm{C} 1 \mathrm{~s}$ and $\mathrm{N} 1 \mathrm{~s}$.
To complement the characterization of the nanoconjugates, TEM investigations were employed to study their morphology. On one hand, detailed analyses of pristine SWCNT revealed mostly the presence of SWCNT bundles. SWCNT-1 and ${ }_{50}$ SWCNT-2, on the other hand, appear with larger diameter, and smaller bundles that coexist with the presence of individual SWCNT, as a result of the effective functionalization (Figure S12). This finding is in line with the increase in intensity of the D-mode in the Raman spectra (Figure S3). On the other hand, the ${ }_{55}$ general aspects of the pristine and functionalized MWCNT are similar in terms of diameter and length (Figure S13).

Graphite exfoliation up to micrometer-sized graphene flakes with thickness of bi-or tri-layer graphene was supported by TEM microscopic analysis (Figure S14). However, reaggregation of ${ }_{60}$ graphene flakes prior to functionalization was only partially prevented by keeping the sample in solution, since GR-2 samples reveal few-layer graphene sheets, which appear crumbled and intertwined (Figure 5).
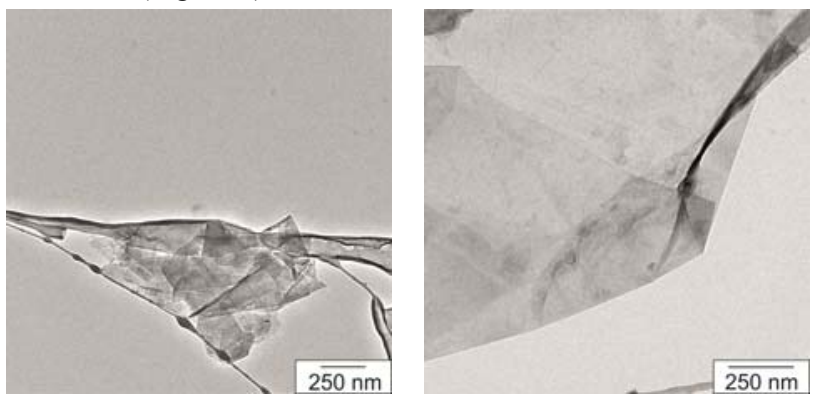

Figure 5. TEM images of GR-2 drop casted from a DMF dispersion onto 65 a lacey carbon grid.

The electrochemical properties of SWCNT-2, MWCNT-2 and GR-2 materials were studied by cyclic voltammetry (CV) and compared with a reference sample of exTTF 2 . In all samples, the single two-electron and chemically quasi-reversible oxidation of 70 the exTTF unit to form dicationic species is clearly discernible. ${ }^{11}$ The oxidations are noticeably broader and positively shifted by 220-270 $\mathrm{mV}$ relative to the reference system 2 (Figure 6), which is likely to be a result of the intramolecular electronic interaction between the electroactive units (CNF and exTTF) in the 75 nanoconjugates, as previously observed in related samples. ${ }^{13 a, b, 29}$

To further evaluate the impact of the covalent functionalization on the electronic properties of the carbon nanoforms synthesized and to investigate the possibility of the formation of radical ion pairs that include the oxidation of exTTF, and the reduction of the 80 different carbon nanoforms, we employed absorption, emission, and transient absorption spectroscopy.

When different SWCNT, SWCNT-1 and SWCNT-2 suspension in $N, N$-dimethylformamide (DMF) were investigated by absorption spectroscopy, in line with previous studies, ${ }^{30}$ the 85 absorption features of covalently functionalized SWCNT are notably blue shifted when compared to the pristine sample (Figure 7). In particular, SWCNT reveals absorption maxima at 560, 661, 741, 814, 1006, 1072, 1172, and $1313 \mathrm{~nm}$. After SWCNT functionalization, the transitions at higher energies in, 90 for example, SWCNT-1 undergo slight hypsochromic shifts to $558,652,733$, and $812 \mathrm{~nm}$, while those at lower energies are shifted appreciably stronger, that is, to $989,1051,1145$, and 1279 $\mathrm{nm}$. The absorption spectrum of SWCNT-2 reveals only a minor 
shift to $557,655,734,810,998,1054,1154$, and $1287 \mathrm{~nm}$. This is likely due to a redistribution of charge density within the covalently functionalized SWCNT.

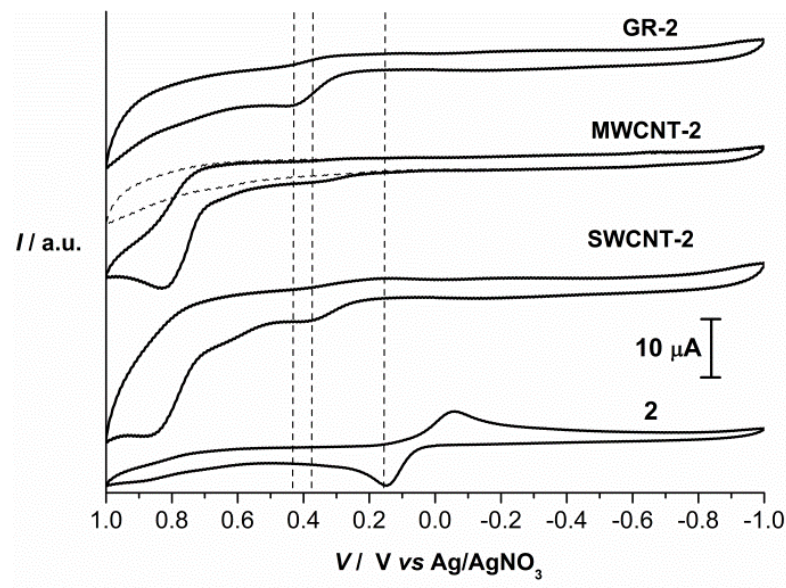

5 Figure 6. Cyclic voltammograms of SWCNT-2, MWCNT-2, GR-2 and reference exTTF 2, obtained in DMF solutions containing $0.1 \mathrm{M} \mathrm{TBAPF}_{6}$ and using $\mathrm{Ag} / \mathrm{AgNO}_{3}$ as reference electrode, glassy carbon as working electrode and a Pt wire counter electrode. Scan rate is $100 \mathrm{mV} / \mathrm{s}$. The dashed line corresponds to the background signal.

10 In addition to the absorption assays, we subjected pristine SWCNTs, SWCNT-1 and SWCNT-2 to fluorescence measurements in DMF. Accordingly, measurements performed with pristine SWCNTs reveal maxima at 1057, 1128, 1211, 1305, and $1419 \mathrm{~nm}$, which correspond to $(10,2),(7,6),(11,3),(13,2)$, 15 and $(9,8)$ SWCNT, respectively (Figure 8$).{ }^{31}$ It is important to note that the fluorescence features mirror image those seen in the absorption measurements. For SWCNT-1 and SWCNT-2, a similar fluorescence pattern was found with just minor hypsochromic shifts relative to pristine SWCNT (Figure 8).

20 Moreover, the comparison between the fluorescence of the functionalized SWCNT with that of pristine SWCNT at equal absorbances at the excitation wavelength, as shown in Figure 9, unravels quenching of the SWCNT fluorescent features. As a matter of fact, the fluorescence intensities of SWCNT-1 and

${ }_{25}$ SWCNT-2 are quenched, with values of 82 and $63 \%$, respectively, relative to pristine SWCNT. While the fluorescence quenching between SWCNT and SWCNT-1 is due to functionalization, the quenching, which relates to a comparison between SWCNT and SWCNT-2, has the additional contribution 30 from an energy and/or electron transfer.

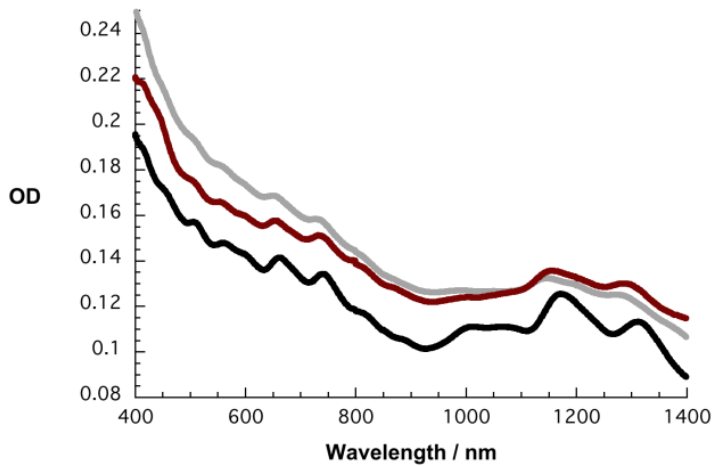

Figure 7. Absorption spectra of SWCNT (black), SWCNT-1 (grey), and SWCNT-2 (red) in DMF.
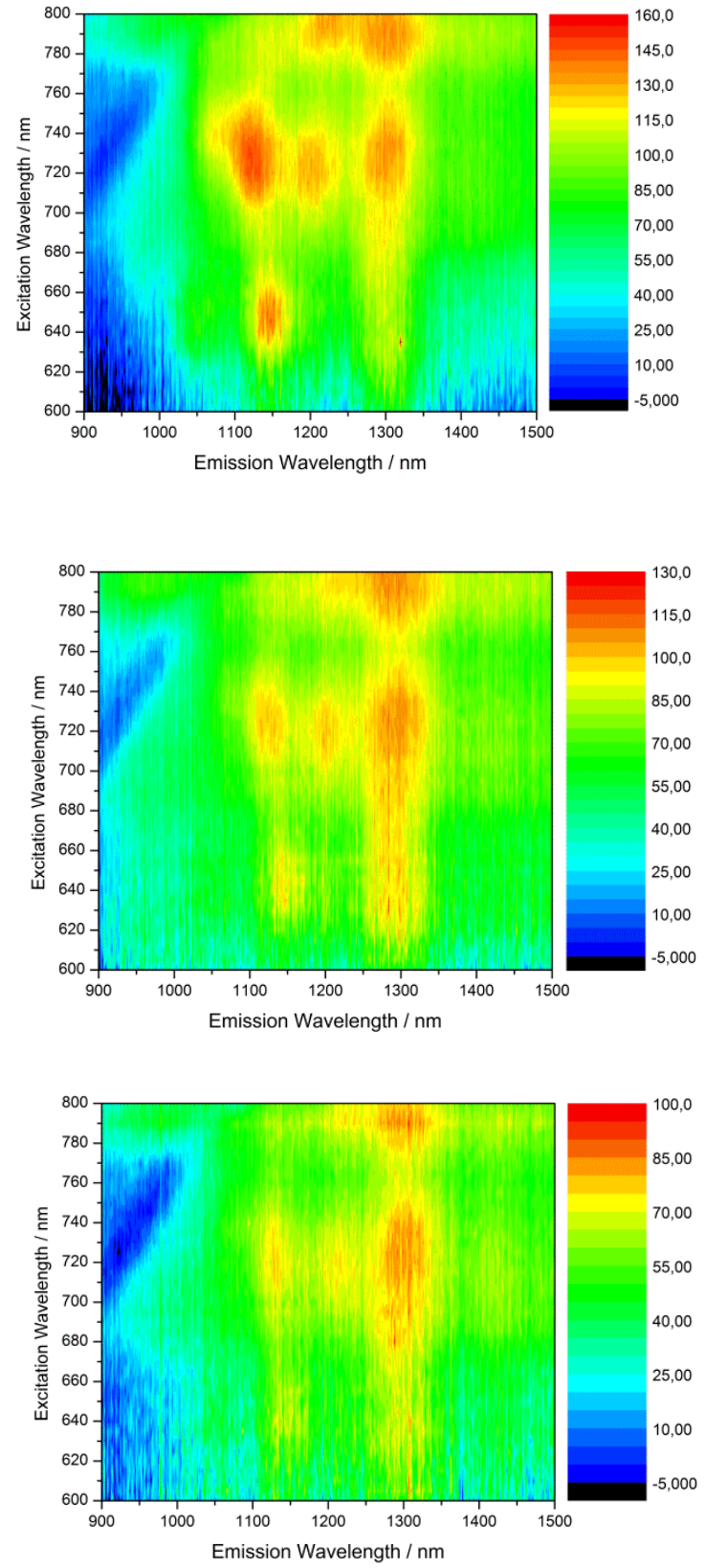

Figure 8. 3D steady-state fluorescence spectra of pristine SWCNT (top), SWCNT-1 (middle), and SWCNT-2 (bottom) in DMF with increasing intensity from blue to green to yellow and to red.

40 Strong support for excited state interactions, that is, between exTTF as electron donor and SWCNT as excited state electron acceptor came from femtosecond transient absorption measurements. Looking at SWCNT, which were simply suspended in DMF, a set of transient minima were observed at 45 455, 515, 565, 570, 665, 1195, 1315, and $1375 \mathrm{~nm}$ (Figure S15). In other words, a negative imprint of the van Hove singularities evolves. As Figure S15 shows, all features decay similarly during the recovery of the ground state with two major lifetime components (i.e., 1.5 and $80 \mathrm{ps}$ ). Overall, no particular shifts of 50 the transitions were observable during the decay. Notably, this behavior resembles that seen numerously for pristine SWCNT suspended in various solvents. When turning to SWCNT-1, minima at $455,510,560,570,660,1190,1310$, and $1360 \mathrm{~nm}$ 
were noted upon $387 \mathrm{~nm}$ excitation (Figure S16). The features are hypsochromically shifted when compared to pristine SWCNT - a finding that reflects the influence noted in the ground state absorption. From a multiwavelength analysis we derive major 5 lifetime components for the excited state decay with values of 1.5 and 65 ps. The overall difference in terms of lifetimes relative to pristine SWCNT relates to the impact that the functionalization exerts on the electron properties of SWCNT. Finally, for SWCNT-2 we note the same minima at 455, 510, 560, 570, 660, 101185,1310 , and $1360 \mathrm{~nm}$ at the conclusion of the excitation (Figure 10). Unlike what it is seen for SWCNT-1, in which minima decay with 1.5 and 65 ps, in SWCNT-2 a major decay takes place with a lifetime of 15 ps. Overall, appreciable blue shifts of the transient bleaching with minima at 500, 554, 650,

151175 and $1360 \mathrm{~nm}$ evolve in this deactivation. Implicit are new conduction bands, in which electrons are injected from exTTF shifting the transitions to lower energies. Important is also the 600 to $800 \mathrm{~nm}$ range, where a new transition correlates well with the one-electron oxidized exTTF. ${ }^{32}$ In other words, the lifetime of

2015 ps in SWCNT-2 correlates with a charge separation. A global analysis resulted in a lifetime of this newly generated radical ion pair state of about $250 \mathrm{ps}$.

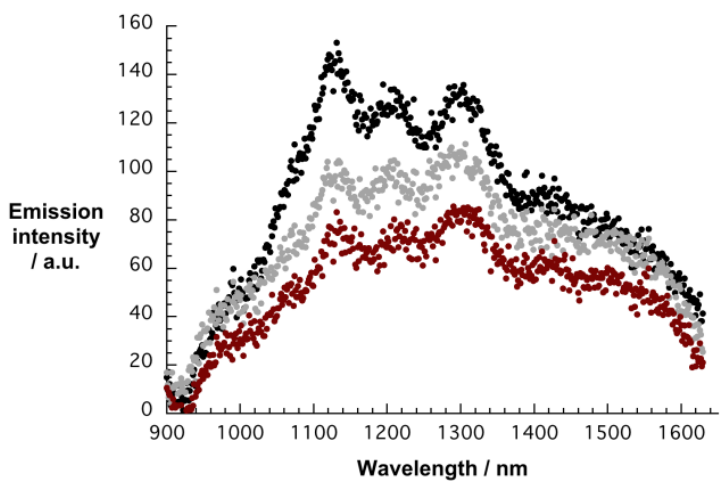

Figure 9. Comparison of the NIR fluorescence spectra of pristine ${ }_{25}$ SWCNT (black), SWCNT-1 (grey) and SWCNT-2 (red) in DMF - 720 $\mathrm{nm}$ excitation.

Unfortunately, the lower degree of functionalization achieved in MWCNT and graphene samples do not allowed a proper photophysical characterization of the aggregates formed. As a 30 matter of fact, following photoexcitation at $387 \mathrm{~nm}$, the differential absorption spectra of GR-2 are like those seen for the starting material, that is, exfoliated graphite (Figure S17). In the visible, we note an ultrashort lived transient - in the form of bleaching - between 550 and $800 \mathrm{~nm}$. This transient transforms 35 within 0.5 ps to a featureless and broad transient. In the nearinfrared, a distinct bleaching evolves between 800 and $1400 \mathrm{~nm}$ and beyond. It is formed with approximately $0.5 \mathrm{ps}$ and can be assigned to a graphite-related phonon bleaching. A closer look at the kinetics at, for example, 625 and $1100 \mathrm{~nm}$ reveals that the 40 transformation of the earlier is linked to the formation of the latter. Nevertheless, both decay monoexponentially to reinstate the baseline. From the lack of any metastable transient, on one hand, and that neither spectroscopic nor kinetic differences emerge relative to exfoliated graphite, on the other hand, we ${ }_{45}$ conclude the absence of electron transfer in photoexcited GR-2.
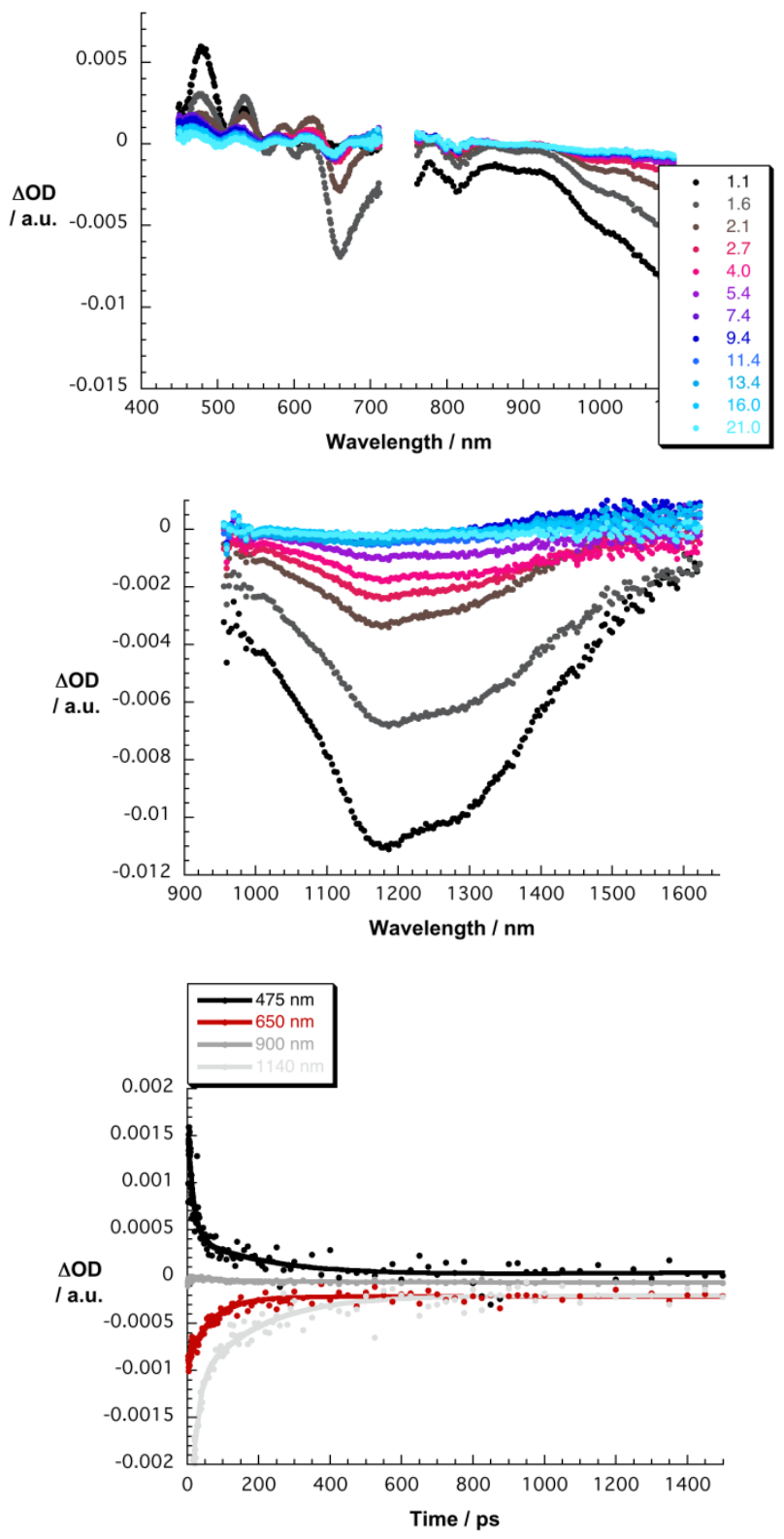

50 Figure 10. Upper part - differential absorption spectra (visible and nearinfrared) obtained upon femtosecond pump probe experiments $(387 \mathrm{~nm})$ of SWCNT-2 with several time delays between 1.1 and 21.0 ps at room temperature. Central part - differential absorption spectra (extended nearinfrared) obtained upon femtosecond pump probe experiments $(387 \mathrm{~nm})$ 55 of SWCNT-2 with several time delays between 1.1 and 21.0 ps at room temperature. Lower part - time absorption profiles of the spectra shown in the upper part at 475, 650, 900, and $1140 \mathrm{~nm}$ monitoring the charge separation and charge recombination.

\section{Conclusions}

${ }_{60} \mathrm{We}$ presented here a comprehensive assay on the covalent functionalization of carbon nanoforms by means of the wellestablished CuAAC reaction. To this end, phenylethinyldecorated SWCNTs, MWCNTs, and graphene were prepared by arylation with diazonium salts under mild conditions in very good ${ }_{65}$ yields. All intermediates and materials were characterized by FTIR, Raman spectroscopy, TGA, and XPS analysis as well as by 
TEM microscopy.

Furthermore, by this covalent method, we have functionalized SWCNTs, MWCNTs and graphene with an electroactive molecule, namely i.e. exTTF, which is evidently observed in the 5 solution electrochemistry performed with all the materials. For SWCNT-2, where the density of exTTFs on the surface was the highest ( 1 per $72 \mathrm{C}$ atoms), a new transition absorption maximum around 600 to $800 \mathrm{~nm}$ range is observed upon photoexcitation. Owing to the fact that the latter is an unambiguous attribute of the 10 one-electron oxidized exTTF it documents the successful formation of the charge separated state.

\section{Acknowledgements}

Financial support from the European Research Council ERC2012-ADG_20120216 (Chirallcarbon), MINECO of Spain (grant 15 numbers CTQ2011-24652 and PIB2010JP-00196), MOLESCO (grant number 606728), and CAM (grant number MADRISOLAR-2 S2009/PPQ-1533) is greatly appreciated. N. M. is indebted to Alexander von Humboldt Foundation. We wish to thank Prof. Philippe Serp for providing us with MWCNT 20 synthesized by the CVD method developed in his laboratory. M. M. O. acknowledges financial support from the Marie Curie COFUND programme "U-Mobility" co-financed by Universidad de Malaga and the European Community's Seventh Framework Programme under Grant Agreement No. 246550.

\section{${ }_{25}$ Notes and references}

a Departamento de Química Orgánica I, Facultad de Química, Universidad Complutense, E-28040 Madrid, Spain. E-mail: maherran@quim.ucm.es,nazmar@quim.ucm.es

${ }^{b}$ Friedrich-Alexander-Universität Erlangen-Nürnberg, Department of ${ }_{30}$ Chemistry and Pharmacy \& Interdisciplinary Center for Molecular Materials (ICMM), Egerlandstrasse 3, 91058 Erlangen, Germany. Email: guldi@chemie.uni-erlangen.de

Current address: Organisch-Chemisches Institut, University of Heidelberg, Im Neuenheimer Feld 270, 69120 Heidelberg, Germany.

${ }_{35}^{d}$ IMDEA-Nanociencia, c/Faraday 9, Campus Cantoblanco, 28049 Madrid, Spain.

$\dagger$ Electronic Supplementary Information (ESI) available: Experimental section, synthetic procedures, characterization data and selected supplementary figures.See DOI: 10.1039/b000000x/

$40 \pm$ Dedicated to Professor Fernando Langa in celebration of his $60^{\text {th }}$ birthday.

$\S$ J. M.-G., L. R.-P. and M. M.-O. equally contributed to this article.

1. a) M. Sharon, M. Sharon, Carbon Nano Forms and Applications, McGraw-Hill, New York, 2010; b) D. M. Guldi, N. Martín, Carbon Nanotubes and Related Structures, Wiley-VCH, Weinheim, 2010; c) T. Akasaka, F. Wudl, S. Nagase, Chemistry of Nanocarbons, John Wiley\&Sons, Chichester, 2010.

2. Special issues devoted to Graphene Chemistry, Properties and 50 Application on Acc. Chem. Res., 2013, 46, 1 (pp. 1-189) and 10 (pp. 2191-2339).

3. H. W. Kroto, J. R. Heath, S. C. O’Brien, R. F. Curl, R. E. Smalley, E. Nature 1985, 318, 162.

4. a) S. Iijima, Nature, 1991, 354, 56; b) S. Iijima, T. Ichihashi, Nature, 1993, 363, 603; c) D. S. Bethune, C. H. Klang, M. S. de Vries, G. Gorman, R. Savoy, J. Vasquez, R. Beyers, Nature, 1993, 363, 605.

5. For reviews, see: a) D. Tasis, N. Tagmatarchis, A. Bianco, M. Prato, Chem. Rev., 2006, 106, 1105; b) Y.-L. Zhao, J. F. Stoddart, Acc. Chem. Res., 2009, 42, 1161; c) P. Singh, S. Campidelli, S. Giordani,

60 D. Bonifazi, A. Bianco, M. Prato, Chem. Soc. Rev., 2009, 38, 2214; d) S. A. Hodge, M. K. Bayazit, K. S. Coleman, M. S. P. Shaffer, Chem. Soc. Rev., 2012, 41, 4409; e) L. Rodríguez-Pérez, M. A.
Herranz, N. Martín, Chem. Commun., 2013, 49, 3721; f) K. Dirian, M. A. Herranz, G. Katsukis, J. Malig, L. Rodríguez-Pérez, C. Romero-Nieto, V. Strauss, N. Martín, D. M. Guldi, Chem. Sci., 2013, 4, 4335; g) E. Vázquez, F. Giacalone, M. Prato, Chem. Soc. Rev., $2014,43,58$.

6. N. Komatsu, F. Wang, Materials, 2010, 3, 3818.

7. F. Wang, T. M. Swager, J. Am. Chem. Soc., 2011, 133, 11181.

70 8. A. Hirsch, O. Vostrowsky Top. Curr. Chem. 2005, 245, 193.

9. a) M.-Q. Yang, N. Zhang, Y.-J. Xu, ACS Appl. Mater. Interfaces, 2013, 5, 1156; b) N. Zhang, Y. Zhang, M.-Q. Yang, Z.-R. Tang, Y.-J. $\mathrm{Xu}$, Journal of Catalysis, 2013, 299, 210; c) M.-E. Ragoussi, S. Casado, R. Ribeiro-Viana, G. de la Torre, J. Rojo, T. Torres, Chem. 75 Sci., 2013, 4, 4035; d) M.-E. Ragoussi, G. Katsukis, A. Roth, J. Malig, G. de la Torre, D. M. Guldi, T. Torres, J. Am. Chem. Soc., 2014, 136, 4593.

10. a) G. Clavè, S. Campidelli, Chem. Sci., 2011, 2, 1887; b) H.-X. Wang, K.-G. Zhou, Y.-L. Xie, J. Zeng, N.-N. Chai, J. Li, H.-L. 80 Zhang, Chem. Commun., 2011, 47, 5747; c) G. Tuci, C. Vinattieri, L. Luconi, M. Ceppatelli, S. Cicchi, A. Brandi, J. Filippi, M. Melucci, G. Giambastiani, Chem. Eur. J., 2012, 18, 8454; d) I. Hijazi, B. Jousselme, P. Jégou, A. Filoramo, S. Campidelli, J. Mater. Chem., 2012, 22, 20936; e) M. Castelaín, G. Martínez, C. Marco, G. Ellis, H. 85 J. Salavagione, Macromolecules, 2013, 46, 8980; f) S. G. Yenchalwar; R. R. Devarapalli, A. B. Deshmukh, M. V. Shelke, Chem. Eur. J., 2014, 20, 7402.

11. F. G. Brunetti, J. L. López, C. Atienza, N. Martín, J. Mater. Chem., 2012, 22, 4188 .

90 12. For examples with fullerenes: a) N. Martín, L. Sánchez, M. A. Herranz, B. M. Illescas, D. M. Guldi, Acc. Chem. Res., 2007, 40, 1015; b) S. Castellanos, A. A. Vieira, B. M. Illescas, V. Sacchetti, C. Schubert, J. Moreno, D. M. Guldi, S. Hecht, N. Martín, Angew. Chem. Int. Ed., 2013, 52, 13985; c) M. Wielopolski, A. MolinaOntoria, C. Schubert, J. T. Margraf, E. Krokos, J. Kirschner, A. Gouloumis, T. Clark, D. M. Guldi, N. Martín, J. Am. Chem. Soc., 2013, 135, 10372.

13. For examples with CNT: a) M. A. Herranz, N. Martín, S. Campidelli, M. Prato, G. Brehm, D. M. Guldi, Angew. Chem. Int. Ed., 2006, 45, 4478 ; b) M. A. Herranz, C. Ehli, S. Campidelli, M. Gutiérrez, G. L. Hug, K. Ohkubo, S. Fukuzumi, M. Prato, N. Martín, D. M. Guldi, J. Am. Chem. Soc., 2008, 130, 66; c) C. Romero-Nieto, R. García, M. A. Herranz, C. Ehli, M. Ruppert, A. Hirsch, D. M. Guldi, N. Martín, J. Am. Chem. Soc., 2012, 134, 9183; d) F. G. Brunetti, C. RomeroNieto, J. López-Andarias, C. Atienza, J. L. López, D. M. Guldi, N. Martín, Angew. Chem. Int. Ed., 2013, 52, 2180; e) J. López-Andarias, J. L. López, C. Atienza, F. G. Brunetti, C. Romero-Nieto, D. M. Guldi, N. Martín, N. Nat. Commun., 2014, 5, 3763.

14. S. González, N. Martín, A. Swartz, D. M. Guldi, Org. Lett., 2003, 5, 557.

15. J. L. Bahr, J. Yang, D. V. Kosynkin, M. J. Bronikowski, R. E. Smalley, J. M. Tour, J. Am. Chem. Soc., 2001, 123, 6536.

16. B. K. Price, J. M. Tour, J. Am. Chem. Soc., 2006, 128, 12899.

17. Y. Hernandez, V. Nicolosi, M. Lotya, F. M. Blighe, Z. Sun, S. De, I. T. McGovern, B. Holland, M. Byrne, Y. K. Gun'Ko, J. J. Boland, P. Niraj, G. Duesberg, S. Krishnamurthy, R. Goodhue, J. Hutchison, V. Scardaci, A. C. Ferrari, J. N. Coleman, Nat. Nanotechnol., 2008, 3, 563.

18. E. Pretsch, P. Buhlmann, M. Badertscher, Structure Determination of Organic Compounds, Springer, Berlin and Heidelberg, 2009.

19. The shifts and broadness observed in the skeletal in-plane vibrations of the different carbon nanoforms are due to different contributions: changes of the interplane bonding, disorder and finite size effects, or bending of the graphitic sheets. See for example: J. Kastner, T. Pichler, H. Kuzmany, S. Curran, W. Blau, D. N. Weldon, M. Delamesiere, S. Draper, H. Zandbergen, Chem. Phys. Lett., 1994, 221, 53.

20. a) R. Graupner, J. Raman Spectrosc., 2007, 38, 673; b) M. S. Dresselhaus, G. Dresselhaus, A. Jorio, A. G. Souza Filho, M. A. Pimenta, R. Saito, Acc. Chem. Res., 2002, 35, 1070.

21. A. de Juan, Y. Pouillon, L. Ruiz-González, A. Torres-Pardo, S. Casado, N. Martín, A. Rubio, E. M. Pérez, Angew. Chem. Int. Ed., 2014, 53, 5394. 
22. L. M. Malard, M. A. Pimenta, G. Dresselhaus, M. S. Dresselhaus, Phys. Rep., 2009, 473, 51.

23. M. S. Dresselhaus, A. Jorio, M. Hofmann, G. Dresselhaus, R. Saito, Nano Lett., 2010, 10, 751.

5 24. A. C. Ferrari, J. C. Meyer, V. Scardaci, C. Casiraghi, M. Lazzeri, F. Mauri, S. Piscanec, D. Jiang, K. S. Novoselov, S. Roth, A. K. Geim, Phys. Rev. Lett., 2006, 97, 187401.

25. In the case of MWCNT and graphene samples, the estimation of rea surface functionalization is less accurate considering the fact that the inner layers remain unfunctionalized.

26. M. E. Lipinska, S. L. H. Rebelo, M F. R. Pereira, J. A. N. F. Gomes, C. Freire, J. L. Figueiredo, Carbon, 2012, 50, 3280.
27. S. Ciampi, T. Böcking, K. A. Kilian, M. James, J. B. Harper, J. J. Gooding, Langmuir, 2007, 23, 9320.

15 28. J. P. Collman, N. K. Devaraj, T. P. A. Eberspacher, C. E. Chidsey, E. D. Langmuir, 2006, 22, 2457.

29. S. P. Economopoulos, G. Rotas, Y. Miyata, H. Shinohara, N. Tagmatarchis, ACS Nano, 2010, 4, 7499.

30. B. Gebhardt, Z. Syrgiannis, C. Backes, R. Graupner, F. Hauke, A. Hirsch, J. Am. Chem. Soc., 2011, 133, 7985.

31. S. M. Bachilo, M. S. Strano, C. Kittrell, R. H. Hauge, R. E. Smalley, R. B. Weisman, Science, 2002, 298, 2361.

32. D. M. Guldi, L. Sánchez, N. Martín, J. Phys. Chem. B, 2001, 105, 7139 


\section{Table of contents entry}

The different reactivity of carbon nanoforms such as SWCNT, MWCNT and graphene involving ${ }_{s}$ sequential arylation and click chemistry reactions has important consequences on the properties of the nanoconjugates obtained with electroactive units.

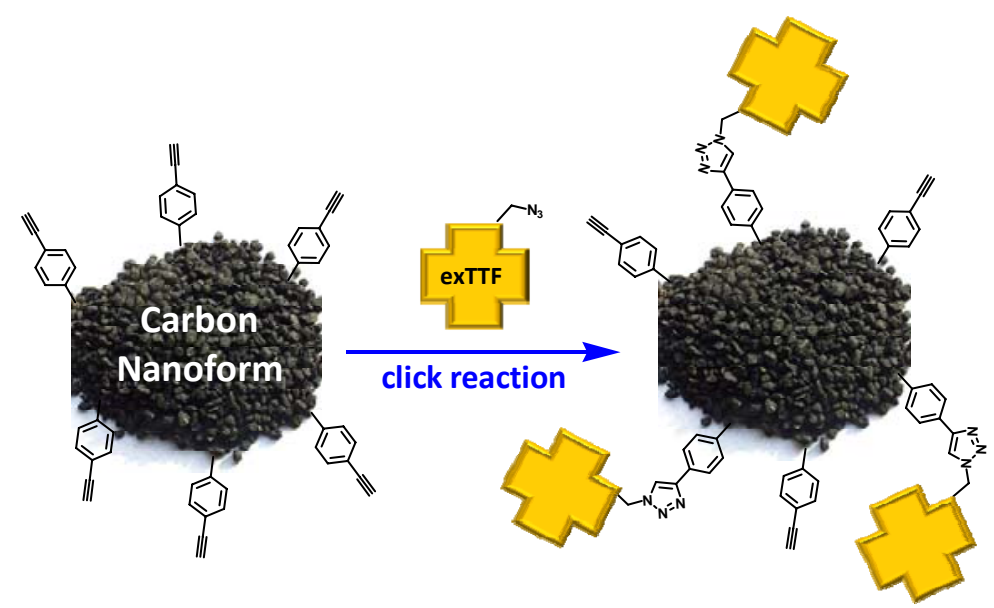

